\title{
Reduced-feedback linear precoding with stable performance for the time-varying MIMO broadcast channel
}

Michael A. Jensen

jensen@byu.edu

Adam L. Anderson

James R. Zeidler

Follow this and additional works at: https://scholarsarchive.byu.edu/facpub

Part of the Electrical and Computer Engineering Commons

\section{Original Publication Citation}

Anderson, A., J. Zeidler, and M. Jensen. "Reduced-Feedback Linear Precoding with Stable Performance for the Time-Varying MIMO Broadcast Channel." Selected Areas in Communications, IEEE Journal on 26.8 (28): 1483-93

\section{BYU ScholarsArchive Citation}

Jensen, Michael A.; Anderson, Adam L.; and Zeidler, James R., "Reduced-feedback linear precoding with stable performance for the time-varying MIMO broadcast channel" (2008). Faculty Publications. 163. https://scholarsarchive.byu.edu/facpub/163 accepted for inclusion in Faculty Publications by an authorized administrator of BYU ScholarsArchive. For more information, please contact ellen_amatangelo@byu.edu. 


\title{
Reduced-Feedback Linear Precoding with Stable Performance for the Time-Varying MIMO Broadcast Channel
}

\author{
Adam L. Anderson, Student Member, IEEE, James R. Zeidler, Fellow, IEEE, and \\ Michael A. Jensen, Fellow, IEEE
}

\begin{abstract}
This work explores the performance of a multipleinput multiple-output broadcast channel where both the transmitter and receivers have outdated channel knowledge due to node motion or other time-variations in the communication channel. A performance analysis based on measured channel responses reveals significant throughput degradation for optimal linear and nonlinear precoding strategies unless the channel state information (CSI) is frequently fed back to the transmitter. The paper then develops a linear beamforming precoding strategy based on channel distribution information in the form of a full spatial correlation matrix for each user. This algorithm is shown to provide highly stable communication, with a throughput that is higher than that for optimal precoders operating on outdated CSI, in a time-variant environment, indicating that this approach can operate with significantly reduced feedback frequency. Furthermore, the paper demonstrates the use of the well-known Kronecker and Weichselberger models to parameterize the full correlation matrix to enable further reduction in the amount of feedback data required for implementation of the new beamforming technique.
\end{abstract}

Index Terms-MIMO systems, Broadcast channels, Array signal processing, Time-varying channels, Feedback systems

\section{INTRODUCTION}

$\mathbf{T}$ HE NETWORK throughput enabled by using nodes equipped with multiple-input multiple-output (MIMO) technology in multi-user wireless systems can be dramatically higher than that obtained with single-antenna radios provided that appropriate multi-user MIMO signaling strategies are employed. For the broadcast channel, sum capacity is achieved using nonlinear dirty-paper coding (DPC), a thorough overview of which appears in [1], based on accurate channel state information (CSI) at the transmitter (CSIT) and receiver (CSIR). Alternatively, an algorithm referred to here as regularized channel inversion (RCI) beamforming has been developed that maximizes the broadcast channel sum-rate under the constraint of linear precoding [2], [3].

While these broadcast channel MIMO precoding strategies offer impressive performance potential for multi-user

Manuscript received November 2, 2007; revised April 14, 2008. This work was sponsored by the U. S. Army Research Office under the Multi-University Research Initiative (MURI) Grant \# W911NF-04-1-0224.

Adam Anderson and Dr. James Zeidler are with the University of California, San Diego Department of Electrical and Computer Engineering La Jolla, CA 92093-0407 (e-mail: a3anders@ucsd.edu, zeidler@ece.ucsd.edu).

Dr. Michael Jensen is with the Brigham Young University Department of Electrical and Computer Engineering Provo, UT 84602 (e-mail: jensen@ee.byu.edu).

Digital Object Identifier 10.1109/JSAC.2008.081014. networks, the resulting sum-rate performance can suffer in time-varying environments where CSI estimates fed back to the transmitter for use in precoder construction can quickly become outdated. In prior work, we have quantified this degradation using both modeled and measured time-variant multi-user channels (assuming perfect CSIR but outdated CSIT), demonstrating that the performance can be reduced to less than half of the optimal value if the nodes move approximately one-half of a wavelength at the communication center frequency [4]. This means that robust performance requires either frequent feedback or the implementation of precoding strategies offering stable performance with channel variations without the use of CSI itself.

One approach for mitigating these difficulties in both singleand multi-antenna channels is to change the type or amount of information used to construct the precoder [5]. For example, a simple method of limiting the quantity of feedback, often referred to as opportunistic or random beamforming [6], [7], is for each user to simply return information regarding the signalto-interference plus noise ratio (SINR) to the transmitter who can then determine user selection for the given beamforming vectors and scheduling algorithm. Variations on and comparisons to opportunistic or random beamforming can be found in [8], [9]. Another approach to limited feedback is to create quantized beamforming codebooks for either single-user [10] or multi-user [11], [12] channels, which when known to transmitter and receiver(s), can reduce the feedback to simply an index into the predetermined codebook. Other methods of feedback quantization are also possible, as in [13] where feedback of normalized channel vectors is compared with standard beamforming codebooks or when information about the channel itself is quantized and fed back to the transmitter [14][16]. Other types of information, often generically referred to as channel quality information (CQI), can also be used for beamformer construction [17]-[19]. Though each of these methods can dramatically reduce the amount of feedback data required, the frequency of feedback must still remain high to compensate for time variations in the channel.

To limit the frequency of feedback, alternatively stable (yet suboptimal) communication performance may be obtained by signaling based on channel distribution information (CDI), often referred to as partial CSI, in the form of either channel mean (CMI) or covariance (CCI) information in both singleuser and multi-user channels [1], [20], [21]. Combining vari- 
ous forms of partial CSI is also possible [22], [23] where both $\mathrm{CQI}$ and CDI are used to perform coarse channel acquisition for scheduling purposes in the broadcast channel. The work in [4] develops a signaling approach for the broadcast channel based on minimum mean squared error (MMSE) beamforming weights at the transmitter and receiver computed from CDI in the form of one-sided spatial correlation matrices under the assumption that the receiver possesses perfect CSIR. The algorithm is shown to provide stable communication performance for time-variant channels with low required feedback frequency.

The goal of this work is to expand on this use of CDI in the time-variant, multi-user MIMO broadcast channel by considering the use of CDI at both the transmitter (CDIT) and receiver (CDIR) to reduce the frequency of channel training, estimation, and feedback. The paper first quantifies the loss in rate resulting from outdated channel knowledge at both the transmitter and receiver and then develops the CDIbased beamforming approach which is an augmentation of the RCI algorithm [2], [3]. Simulations of the algorithm using realistic multi-user MIMO channels measured in both indoor and outdoor environments [24], [25] demonstrate that the resulting stable communication performance exceeds that of DPC or RCI for reduced feedback frequency. The fact that this performance is obtained in measured channels emphasizes that the approach is not tied to specific assumptions about channel behavior that might be embedded in typical channel modeling strategies. The drawback of the algorithm, however, is that since it requires the full spatial covariance matrix, each user must feed back a significant amount of information to describe the spatial correlation compared to CSI-based schemes. The paper therefore develops a method for parameterizing the required CDI using two commonly accepted channel models, namely the Kronecker [26] and Weichselberger [27] models, that can both be utilized to explicitly represent the spatial covariance structure. Simulations in realistic channels show that this parameterization reduces the feedback on par with CSI-based schemes with minor loss in throughput performance while significantly lowering the frequency of feedback.

Throughout the analysis, scalars are written in lower-case, while vectors and matrices are written as bold-face in loweror upper-case, respectively. The meaning of subscripts on matrices depends on the type of matrix being referenced. For matrices that are unique to each user in the network, the subscript reflects that user (e.g. $\mathbf{A}_{i}$ is the $\mathbf{A}$ matrix for user $i$ ). When a matrix is shared by all users in the network, then the indices refer to elements within the matrix (e.g. $\mathbf{B}_{i, j}$ is the $j$ th element in the $i$ th row and $\mathbf{B}_{:, j}$ is the $j$ th column of B). Standard matrix operations of transpose, conjugate, and conjugate-transpose are defined as $\{\cdot\}^{T},\{\cdot\}^{*}$, and $\{\cdot\}^{H}$, respectively. The function $\operatorname{vec}(\cdot)$ is the matrix column stacking operator while $\operatorname{mat}(\cdot)$ is its inverse (e.g. $\operatorname{mat}(\operatorname{vec}(\mathbf{A}))=\mathbf{A})$. Finally, $\otimes$ is the matrix Kronecker product while $\odot$ is the Schur-Hadamard or elementwise matrix product.

\section{Time-VARYing MIMO BRoAdCAST ChanNel With OUTDATED CSI}

Fig. 1 shows a diagram of the $K$-user, time-varying, MIMO broadcast channel with virtual feedforward and feedback links representing the conveyance of delayed channel information between the transmitter and receiver. For this work, $K$ independent data streams are precoded at the transmitter using one of several algorithms described in this paper. Since the precoding schemes attempt to maximize the system sum-rate, users with poor channels may be excluded, indicating that some streams may be denied channel access. For precoding purposes, the transmitter has access to all users' channel matrices delayed by $\Delta_{t}$ samples from the current time. This delay will be modeled in this section as either outdated or erroneous CSI. Similarly, during decoding, the receivers possess a channel realization or receive beamforming vector that is delayed $\Delta_{\mathrm{r}}$ samples from the current channel. We emphasize that the feedforward path simply represents a model for accounting for the delay $\Delta_{\mathrm{r}}$, and that the true delay mechanism would be a finite interval between training events combined with network overhead and channel estimation errors. Thus, the feedforward and feedback "channels" as shown in Fig. 1 are simply graphical tools in describing the error introduced in the time-varying MIMO broadcast channel and do not represent any actual implementation scheme. However, given the additional delay for CSIT that would be incurred for feedback, it is logical to assume that $\Delta_{\mathrm{t}} \geq \Delta_{\mathrm{r}}$ with equality in the ideal, instantaneous feedback case. Other than these delays, the channel estimates and feedback communication are considered error-free; the effects of channel estimation error due to limited training is not examined in this work. Based on the availability of measured channel data, we impose the system restrictions that the transmitter and each of the $K \leq 6$ receivers will each be equipped with $M \leq 8$ antennas.

\section{A. Rate Optimal Transmit Precoding}

Nonlinear dirty-paper coding [1] is optimal in the sense that it maximizes the sum mutual information (and therefore sum capacity) when the transmitter and receivers have perfect CSI. DPC is often considered infeasible in practice [3] but will be analyzed as a benchmark for other precoding algorithms.

The impact of channel estimation error in a two-user singleinput single-output (SISO) channel using DPC was analyzed in [28]. Consider the case of DPC in a MIMO channel when user ordering is not optimized at the transmitter, where user 1 is encoded first, user 2 second, and so on. At the transmitter, the DPC algorithm attempts to successively presubtract interference from previously encoded users, while the receiver uses its delayed channel knowledge for detection. In [28] the authors refer to DPC as "naive" when only presubtracting interference with the given channel estimates; this same naive approach to DPC will be used for the MIMO case. As an example, consider the received vector for the first encoded user

$$
\mathbf{y}_{1}=\widetilde{\mathbf{H}}_{1} \mathbf{x}_{1}+\mathbf{E}_{1}\left(\Delta_{\mathrm{r}}\right) \sum_{i=1}^{K} \mathbf{x}_{i}+\widetilde{\mathbf{H}}_{1} \sum_{i=2}^{K} \mathbf{x}_{i}+\boldsymbol{\eta}_{1}
$$

where $\boldsymbol{\eta}_{1}$ is zero-mean, unit-variance additive white Gaussian noise (AWGN). If $\mathbf{H}_{1}$ represents the actual channel to the 1st user and $\hat{\mathbf{H}}_{1}\left(\Delta_{n}\right)$ is outdated CSI used to construct the precoder $\left(\Delta_{n}=\Delta_{\mathrm{t}}\right)$ or decoder $\left(\Delta_{n}=\Delta_{\mathrm{r}}\right)$, then the error $\mathbf{H}_{1}-\widehat{\mathbf{H}}_{1}\left(\Delta_{n}\right)=\mathbf{E}_{\mu, 1}+\mathbf{E}_{1}\left(\Delta_{n}\right)$ where $\mathbf{E}_{\mu, 1}$ is the mean of the error and $\mathbf{E}_{1}\left(\Delta_{n}\right)$ is a zero-mean random matrix. Finally, 


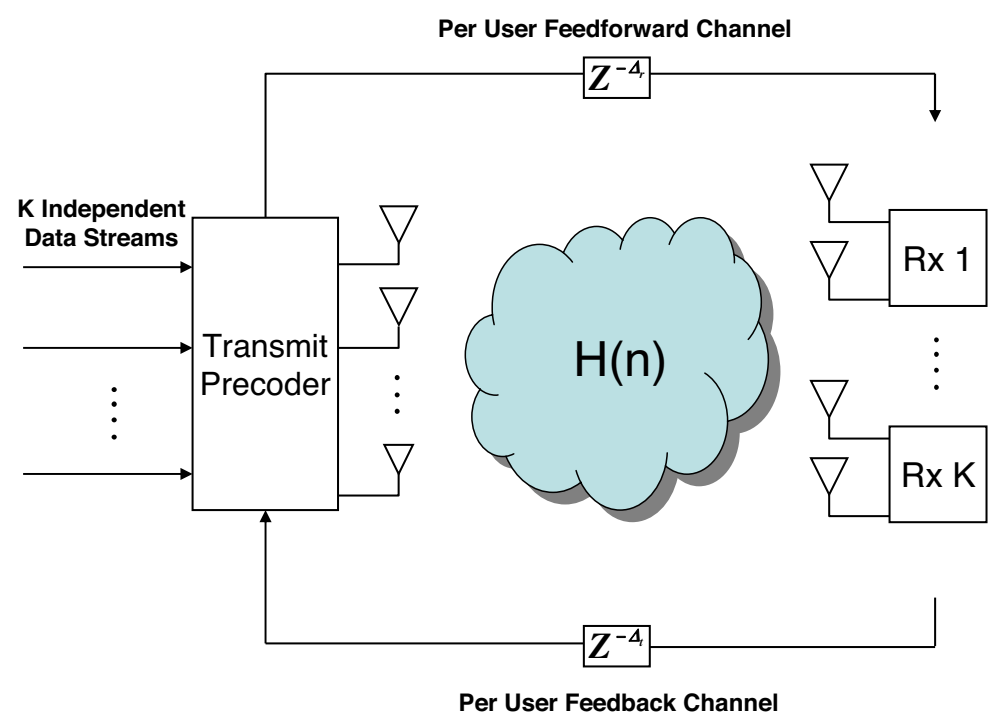

Fig. 1. A K-user MIMO broadcast channel where the transmitter has channel knowledge of all users delayed by $\Delta_{t}$ samples from the current channel and receivers have individual channel knowledge delayed by $\Delta_{r}$ samples from the current channel. All nodes are assumed to have $M$ antennas.

$\widetilde{\mathbf{H}}_{1}=\widehat{\mathbf{H}}_{1}+\mathbf{E}_{\mu, 1}$. Note that the first interference term is caused strictly by having erroneous CSIR while the second interference term is a direct result of being encoded first in the DPC scheme. The received vector for the second user becomes

$$
\begin{aligned}
\mathbf{y}_{2}= & \widetilde{\mathbf{H}}_{2} \mathbf{x}_{2}+\mathbf{E}_{2}\left(\Delta_{\mathrm{r}}\right) \sum_{i=2}^{K} \mathbf{x}_{i} \\
& +\mathbf{E}_{2}\left(\Delta_{\mathrm{t}}\right) \mathbf{x}_{1}+\widetilde{\mathbf{H}}_{2} \sum_{i=3}^{K} \mathbf{x}_{i}+\boldsymbol{\eta}_{2}
\end{aligned}
$$

where the additional interference arises from the transmitter only being able to remove the known portion of the signal encoded for user 1.

Continuing in a similar manner, for a precoded transmit vector $\mathbf{x}_{j}$ destined for the $j$ th user, the resulting receive vector for the $j$ th user can be written as

$$
\begin{aligned}
\mathbf{y}_{j}= & \widetilde{\mathbf{H}}_{j} \mathbf{x}_{j}+\overbrace{\mathbf{E}_{j}\left(\Delta_{\mathrm{r}}\right) \sum_{i=j}^{K} \mathbf{x}_{i}}^{\text {outdated CSIR }}+\overbrace{\mathbf{E}_{j}\left(\Delta_{\mathrm{t}}\right) \sum_{i=1}^{j-1} \mathbf{x}_{i}}^{\text {outdated CSIT }} \\
& +\widetilde{\mathbf{H}}_{j} \sum_{i=j+1}^{K} \mathbf{x}_{i}+\boldsymbol{\eta}_{j} .
\end{aligned}
$$

Since we cannot assume that the transmitter knows any information about $\mathbf{E}_{j}\left(\Delta_{n}\right)$, we consider the worst-case scenario where it is modeled as a zero mean Gaussian matrix whose variance grows with delay $\Delta_{\mathrm{t}}$ or $\Delta_{\mathrm{r}}$. Despite the fact that time indices are dropped for clarity, it is important to recognize that the received vector is a function of the delay in channel feedback. Eq. (3) highlights those portions of the interference in the received signal that are caused by delayed channel knowledge.

The exact mutual information for the transmit and received vectors in (3) is unknown, and therefore the lower bound suggested in [29] will be adapted for use in the broadcast channel to include the additional interference terms shown in Eq. (3). Details of this analysis appear in Appendix A, with the result that the mutual information for user $j$ is bounded by

$$
\begin{aligned}
I_{\mathrm{DPC}}\left(\mathbf{x}_{j} ; \mathbf{y}_{j} \mid \widetilde{\mathbf{H}}_{j}\right) & \geq \log \left|\mathbf{I}+\widetilde{\mathbf{H}}_{j}^{H}\left(\mathbf{I}+\mathbf{Z}_{j}\right)^{-1} \widetilde{\mathbf{H}}_{j} \mathbf{Q}_{j}\right| \\
\mathbf{Z}_{j} & =\boldsymbol{\Psi}_{\mathbf{E}_{\mathrm{r}, j}}^{\sum_{i=j}^{K} \mathbf{Q}_{i}}+\mathbf{\Psi}_{\mathbf{E}_{\mathrm{t}, j}}^{\sum_{i=1}^{j-1} \mathbf{Q}_{i}}+\mathbf{\Psi}_{\widetilde{\mathbf{H}}_{j}}^{\sum_{i>j} \mathbf{Q}_{i}}
\end{aligned}
$$

where $\mathbf{x}_{j}$ are assumed to be Gaussian inputs, the input covariance matrices are given by $\mathbf{Q}_{j}=\mathrm{E}\left[\mathbf{x}_{j} \mathbf{x}_{j}^{H}\right], \mathbf{I}$ is the identity matrix, $\mathbf{\Psi}_{\mathbf{V}}^{\mathbf{U}}=\mathrm{E}\left[\mathbf{V U V}^{H}\right]$ for matrices $\mathbf{U}$ and $\mathbf{V}$, and $\mathbf{E}_{\mathrm{r}, j}=\mathbf{E}_{j}\left(\Delta_{\mathrm{r}}\right)$ and $\mathbf{E}_{\mathrm{t}, j}=\mathbf{E}_{j}\left(\Delta_{\mathrm{t}}\right)$ are used for simplicity as explained in Appendix A. The sum mutual information used for analysis in this work is then

$$
C_{\mathrm{DPC}}\left(\Delta_{\mathrm{r}}, \Delta_{\mathrm{t}}\right)=\sum_{j=1}^{K} I_{\mathrm{DPC}}\left(\mathbf{x}_{j} ; \mathbf{y}_{j} \mid \widetilde{\mathbf{H}}_{j}\right)
$$

where $C_{\mathrm{DPC}}\left(\Delta_{\mathrm{r}}, \Delta_{\mathrm{t}}\right)$ is implicitly a function of the input covariance matrices $\mathbf{Q}_{j}$. For the simulations in this work, the input covariance matrices are found by naively applying iterative water-filling using the duality of the multiple access channel and broadcast channel [1] based on the known portions of CSIT. This implies that when $\Delta_{\mathrm{t}}=\Delta_{\mathrm{r}}=0$ (i.e. no channel error or delay), (5) reduces exactly to the sum capacity of the broadcast channel, but when $\Delta_{\mathrm{t}}, \Delta_{\mathrm{r}} \geq 0$ additional performance loss will be seen in the system due to suboptimal input covariances.

\section{B. Rate Maximizing Linear Precoding}

Linear transmit precoding, or beamforming, uses linear preprocessing to mitigate multi-user interference in an effort to optimize various communication parameters [3]. Because this paper considers techniques which maximize the sum mutual information, we will consider the rate-maximizing RCI technique found in [2], [3]. This algorithm assumes a single 
data stream is transmitted to each user unless the user has been excluded from channel access. Allowing multiple streams per user is straightforward and only adds complexity to the beamforming algorithm; however, for the "square" systems (equal number of transmit and receive antennas) considered in this work, the beamformers almost always choose a single stream per user even when given the option of multiple streams. Including these multiple streams does provide additional performance; however, multi-stream gains are marginal for the channels considered and will therefore be omitted for clarity. The received signal vector for user $j$ is then

$$
\mathbf{y}_{j}=\mathbf{H}_{j} \mathbf{b}_{j}\left(\Delta_{\mathrm{t}}\right) \mathbf{x}_{j}+\mathbf{H}_{j} \sum_{i \neq j}^{K} \mathbf{b}_{i}\left(\Delta_{\mathrm{t}}\right) \mathbf{x}_{i}+\boldsymbol{\eta}_{j}
$$

where $\mathbf{b}_{j}\left(\Delta_{\mathrm{t}}\right)$ is the transmit beamforming vector calculated using the RCI algorithm from the channel knowledge delayed by $\Delta_{t}$ samples. It is assumed that each user only has knowledge of their individual channel matrix and the transmit weights assigned to all streams; thus, receive beamforming weights $\mathbf{w}_{j}\left(\Delta_{\mathrm{r}}\right)$ are calculated at each node using the MMSE criterion based on the channel knowledge delayed by $\Delta_{\mathrm{r}}$ samples and transmit weights delayed by $\Delta_{t}$ samples.

Application of this processing reduces the system to a single stream per user with mutual information given by

$$
I_{\mathrm{BF}}\left(\mathbf{x}_{j} ; \mathbf{y}_{j} \mid \rho_{j}\right)=\log \left(1+\rho_{j}\right)
$$

where

$$
\rho_{j}=\frac{\left|\mathbf{w}_{j}^{H} \mathbf{H}_{j} \mathbf{b}_{j}\right|^{2}}{1+\sum_{i \neq j}\left|\mathbf{w}_{j}^{H} \mathbf{H}_{j} \mathbf{b}_{i}\right|^{2}}
$$

and throughput degradation arises from using outdated beamforming vectors on the current channel. When the RCI algorithm excludes a user from the channel, the weight vector $\mathbf{b}_{i}$ becomes zero and equivalently $\rho_{i}=0$. For completeness, one can write the total expected rate given the outdated beamforming weights as

$$
C_{\mathrm{BF}}=\sum_{j=1}^{K} I_{\mathrm{BF}}\left[\mathbf{x}_{j} ; \mathbf{y}_{j} \mid \rho_{j}\right]
$$

It is shown in [2] that the weights which maximize the sumrate from (9) in a broadcast channel with linear preprocessing have the form

$$
\mathbf{B}=\left(\frac{\operatorname{tr}(\mathbf{D})}{P} \mathbf{I}+\mathbf{H}^{*} \mathbf{D}_{j} \mathbf{H}\right)^{-1} \mathbf{H}^{*} \boldsymbol{\Lambda}
$$

where $\mathbf{B}=\left[\mathbf{b}_{1}, \ldots, \mathbf{b}_{K}\right]$ is the matrix of beamforming weights for each user, $\boldsymbol{\Lambda}$ and $\mathbf{D}$ are diagonal weighting matrices [2], and each row of $\mathbf{H}$ represents the channel for each of the individual MISO channels. An iterative procedure was shown in [2] that guarantees convergence to a local maximum of the sum-rate. Note that the only input parameter to the RCI algorithm is the channel transfer matrix for each user.

Some comments are necessary regarding the capacity maximizing MISO RCI beamforming algorithm. In [3], this technique was used with multiple receive antennas by iteratively performing the algorithm while updating the receiver beamformer with MMSE weights, although no proof of optimality was made. Indeed, varying the initial condition of the diagonal weight vectors can produce different solutions. Since the beamforming weights are, in form, capacity optimal for the MISO channel and have the structure of a regularized channel inversion, it will be used as the rate-maximizing beamformer for the MIMO channel with linear processing.

\section{Channel Measurements and Performance SIMULATIONS}

To assess the loss in rate created by outdated CSIT and CSIR and, perhaps more importantly, to ensure that the beamforming algorithm based on CDIT and CDIR are effective in actual channels and not optimized for specified conditions that are assumed in a generalized channel model, all performance analysis in this paper will be accomplished using channel transfer functions obtained from experimental measurements. The equipment used for these measurements samples a pointto-point MIMO channel with $M=8$ transmit and receive antennas. Specific details of the measurement equipment are available in [30].

Prior to data collection, calibration measurements were taken with the transmitter "off" to measure the level of background interference. At the chosen carrier frequency of $2.45 \mathrm{GHz}$, the external interference was found to be below the receiver noise floor in the environments considered. A second calibration performed with both the transmitter and receiver "on" but stationary revealed that the time variation of the channel caused by ambient changes such as pedestrians, atmospheric conditions, and other natural disturbances was insignificant for the environments examined in this paper.

The channel coefficients used in this analysis were measured with a stationary transmitter and a receiver moving at a constant pedestrian velocity $(30 \mathrm{~cm} / \mathrm{s})$. Since the channel is highly oversampled, with samples taken every 2.5 or $3.2 \mathrm{~ms}$, data decimation or interpolation can be used to create any effective node velocity. After channel acquisition, each individual realization of the channel is normalized to have unit average SISO gain [24]. For a given transmitter location, measurements for different receiver locations were taken (using the same receiver velocity), with each location producing the channel matrix for one user in either (3) or (6) for the simulated multi-user network. Since it was observed that channel time variation results almost exclusively from node movement, the superposition of these asynchronous measurements into a single synchronized multi-user broadcast channel seems reasonable. For simulation purposes, measurements taken from three different environments will be used in this work. The "Urban" dataset was acquired in a locale with large buildings and other mobile and stationary obstructions while "Indoor" contains channels measured inside the fourth floor of the Brigham Young University engineering building. "Outdoor" refers to a typical university campus where the transmitter was specifically located in an area surrounded by trees.

The statistical space-time-frequency structure of the experimental MIMO channels has been well analyzed in the literature [31], [32], with ensemble averages over a variety of locations showing that the coefficients obey a complex Gaussian distribution (Rayleigh channel magnitudes) with spatial 


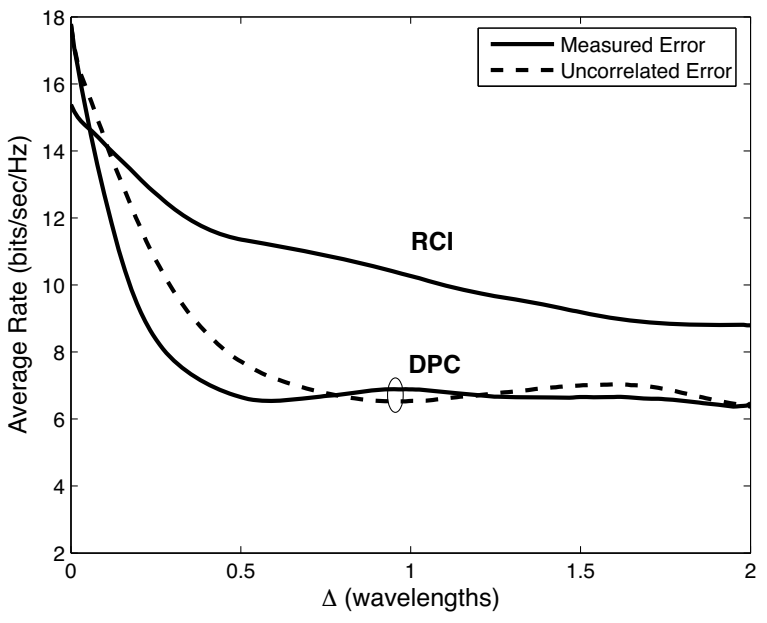

Fig. 2. Average sum-rate of DPC and RCI versus displacement for $M=$ $K=4$ and $P=10$. The receivers have perfect $\operatorname{CSIR}\left(\Delta_{\mathrm{r}}=0\right)$ while the transmitter has delayed knowledge of the channel given by $\Delta$ (wavelengths) from the current channel.

and temporal correlation functions that closely resemble those generated using the classic Jakes' model [24]. Therefore, the measured data will be used to estimate all the parameters necessary to analyze sum-rate performance of the MIMO broadcast channel with delayed feedback as well as input parameters for the given precoding algorithms.

The stochastic quantities of interest are the one-sided transmit and receive spatial correlation matrices, the full spatial correlation matrix, and the mean channel error as well as $\Psi_{\mathbf{E}_{j}}^{\sum_{i} \mathbf{Q}_{i}}$ (see (4)) as a function of delay. The transmit and receive correlation matrices can be estimated using a sample mean based on $N$ samples using

$$
\begin{aligned}
\mathbf{R}_{\mathrm{t}, j} & =\frac{1}{N M} \sum_{n=0}^{N-1} \mathbf{H}_{j}^{T}(n) \mathbf{H}_{j}^{*}(n) \\
\mathbf{R}_{\mathrm{r}, j} & =\frac{1}{N M} \sum_{n=0}^{N-1} \mathbf{H}_{j}(n) \mathbf{H}_{j}^{H}(n) .
\end{aligned}
$$

Similarly, the full $M^{2} \times M^{2}$ spatial covariance matrix for the elements of the channel matrix can be estimated using

$$
\mathbf{R}_{j}=\frac{1}{N M^{2}} \sum_{n=0}^{N-1} \operatorname{vec}\left(\mathbf{H}_{j}(n)\right) \operatorname{vec}\left(\mathbf{H}_{j}(n)\right)^{H} .
$$

Finally, using that $\Psi_{\mathbf{V}}^{\mathrm{U}}=\mathrm{E}\left[\mathbf{V} \mathbf{U V}^{H}\right]=\mathrm{E}\left[\mathbf{V}^{*} \otimes \mathbf{V}\right] \operatorname{vec}(\mathbf{U})$, we can again use a sample mean to construct the estimate

$$
\begin{aligned}
& \mathrm{E}\left[\mathbf{E}_{j}^{*}\left(\Delta_{n}\right) \otimes \mathbf{E}_{j}\left(\Delta_{n}\right)\right]= \\
& \frac{1}{N} \sum_{n=\Delta_{n}}^{N+\Delta_{n}-1}\left(\mathbf{H}_{j}(n)-\mathbf{H}_{j}\left(n-\Delta_{n}\right)\right)^{*} \otimes\left(\mathbf{H}_{j}(n)-\mathbf{H}_{j}\left(n-\Delta_{n}\right)\right)
\end{aligned}
$$

where $\Delta_{n}$ is integer valued. The mean $\mathbf{E}_{\mu, j}$ can be easily estimated from a sample mean of the channel error.

It is important to recognize that as a node moves, the resulting changes in the directions of departure/arrivals of the multipath components result in statistically non-stationary

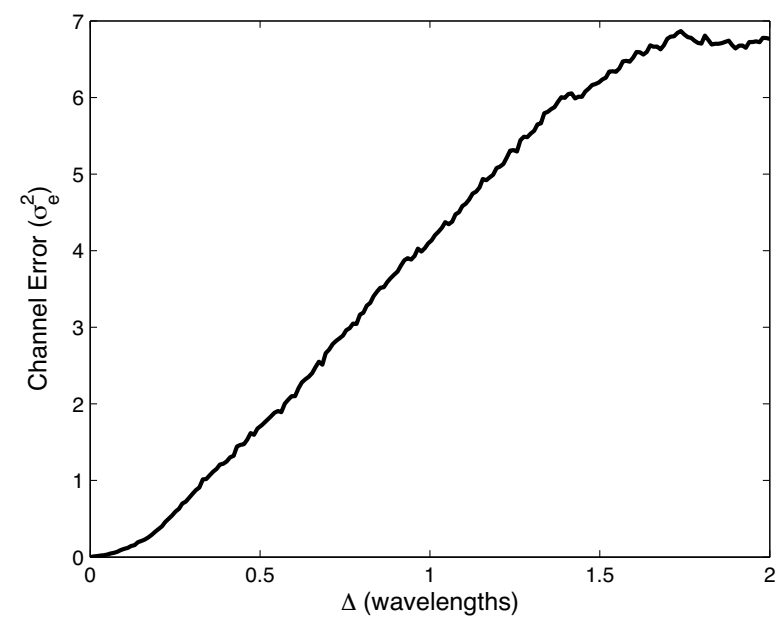

Fig. 3. Channel error variance $\sigma_{e}^{2}$ versus feedback delay as estimated by the measured data.

channel matrices. However, the datasets are partitioned into segments over which the channel remains approximately stationary. This distance, which spans several wavelengths, is much larger than the sub-wavelength coherence distance of the channel.

These estimates of the channel distribution information as a function of delay can be input directly into (5) and (9) to compute the loss in sum rate created by outdated CSI. For these simulations, the datasets are decimated such that each of the $K=4$ users (receivers) as well as the transmitter have $M=4$ antennas. The power is held constant for both precoding techniques such that $\sum_{i=1}^{K} \operatorname{tr}\left(\mathbf{Q}_{i}\right)=\operatorname{tr}(\mathbf{B})=P=10$. The independent variable for the simulations is the physical displacement between the current position and the position at which the channel was measured which is used for detection and precoding, or $\Delta=\Delta_{n} T_{s} v$ where $T_{s}$ and $v$ represent the sample interval of the measured data and the receiver velocity, respectively, and $\Delta_{n}$ is integer valued. This displacement will be expressed in terms of wavelengths at the center frequency of $2.45 \mathrm{GHz}$.

Fig. 2 displays the sum-rate for both DPC and RCI as a function of $\Delta=\Delta_{\mathrm{t}} T_{s} v$ when CSIR is perfect $\left(\Delta_{\mathrm{r}}=0\right)$ in the Urban environment. The plot also shows the rate for DPC when the channel error covariance is a scaled identity (i.e. the channel error is i.i.d.), with the scale factor $\sigma_{e}^{2}$ representing the variance of the error as a function of $\Delta_{\mathrm{t}}$ estimated from the data as shown in Fig. 3. The results in Fig. 2 demonstrate that the difference between correlated and uncorrelated error is relatively small. Furthermore, it is important to recognize that in a real system, the performance degradation will typically be more severe for both precoders since channel estimation errors have been neglected in this study.

Fig. 4 displays the sum-rate for DPC and RCI when both CSIR and CSIT are equally outdated $\left(\Delta_{\mathrm{r}}=\Delta_{t}\right)$ also in the Urban environment. This scenario represents the ideal case when the receiver has erroneous CSI but an instantaneous feedback channel to the transmitter. The performance of both techniques is highly sensitive to the displacement $\Delta$, with the majority of loss relative to the peak performance 


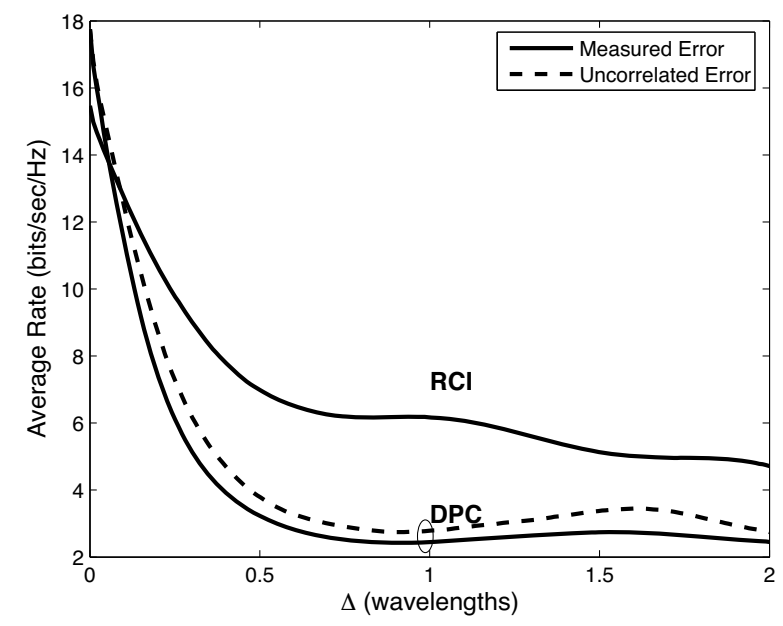

Fig. 4. Average sum-rate of DPC and RCI versus displacement for $M=$ $K=4$ and $P=10$. The receiver and transmitter share equally delayed knowledge of that channel given by $\Delta$ (wavelengths) from the current channel.

occurring before one wavelength of displacement (roughly $12 \mathrm{~cm})$. This severe degradation is not surprising since this displacement is well over the channel coherence distance and the use of outdated CSIR reduces the ability of the receiver to compensate for interference introduced by imperfect CSIT at the transmitter with DPC and receive beamforming vectors are no longer operating on optimal subspaces of the channel with RCI. This high sensitivity for both nonlinear and linear precoding suggests that either frequent, low-error channel estimation and feedback must be available, or the system must employ techniques that may be suboptimal but allow strong performance with reduced feedback frequency.

\section{REgularized CHANNEL Distribution INVERSiON (RCDI) BEAMFORMING}

The severe loss in rate with outdated CSI observed in the prior section motivates the development of new precoding and detection techniques whose performance is less dependent on instantaneous channel state information and more tolerant to infrequent channel estimation and feedback. The problem of achievable throughput given partial CSI is the focus of several recent papers for the single- and multi-user channels (see [1] and references therein). This section will derive a beamforming technique that attempts to maximize the average sum-rate of the MIMO broadcast channel and thereby provides stable overall performance with significant reduction in feedback frequency. This beamformer will only be a function of the CDI.

Unfortunately, formulating the average sum-rate in a form that lends itself to maximization poses difficulties. Therefore, we will use a combination of the approaches outlined in [4] of maximizing the lower bound on an idealized average sum-rate, and [2] of deriving the form of the maximizing beamformer weights. The general steps followed for finding the beamforming weights that maximize the approximate sumrate are: 1) define the sum-rate objective function as a function of the beamforming vectors, 2) solve for the weights that provide maxima to the objective function, and 3) define an
TABLE I

ITERATIVE BEAMFORMING FOR MIMO REGULARIZED CHANNEL DISTRIBUTION INVERSION (RCDI)

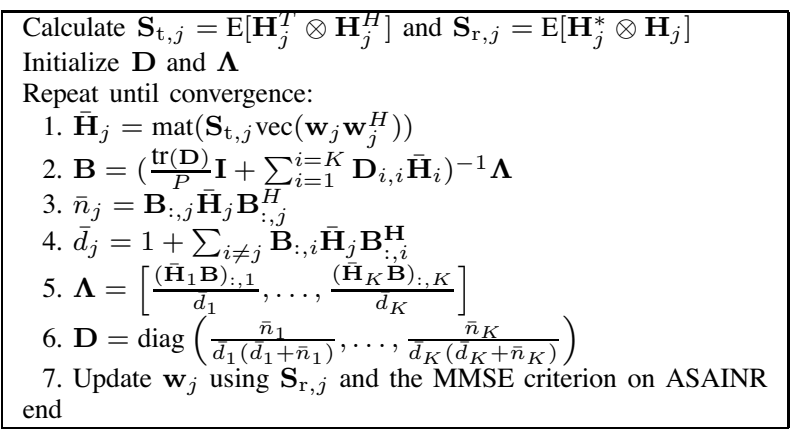

iterative algorithm that finds the weights given some initial condition.

Under the constraint of linear preprocessing, the objective function used can be written as

$$
\bar{C}=\sum_{j=1}^{K} \log \left(1+\frac{\bar{n}_{j}}{\bar{d}_{j}}\right)
$$

where $\bar{n}_{j}=\mathrm{E}\left[\operatorname{num}\left(\rho_{j}\right)\right], \bar{d}_{j}=\mathrm{E}\left[\operatorname{den}\left(\rho_{j}\right)\right]$, and $\operatorname{num}(\cdot)$ and $\operatorname{den}(\cdot)$ return the numerator and denominator of the argument, respectively. The optimization problem is then to construct transmit and receive beamforming vectors to maximize this quantity, or

$$
\max _{\mathbf{w}_{j}, \mathbf{b}_{j}} \bar{C}
$$

with power constraints imposed on the input beamforming vectors.

The details of the maximization process can be found in Appendix B. The resulting regularized channel distribution inversion (RCDI) beamformer matrix $\mathbf{B}$, each column of which represents the transmit beamformer $\mathbf{b}_{j}$ for the $j$ th user, is given as

$$
\mathbf{B}=\left(\frac{\operatorname{tr}(\mathbf{D})}{P} \mathbf{I}+\sum_{i=1}^{K} \mathbf{D}_{i, i} \overline{\mathbf{H}}_{j}\right)^{-1} \boldsymbol{\Lambda}
$$

where the definitions for $\mathbf{D}, \boldsymbol{\Lambda}$, and $\overline{\mathbf{H}}_{j}$ can be found in Appendix B. It is interesting that the form of (16) is similar to that in (10) for the RCI beamformer (see also [2]). Since, however, RCI depends on CSI, the required feedback frequency for RCI is on the order of the channel coherence time. In contrast, since RCDI depends on CDI, feedback is only required when the channel correlation structure changes appreciably.

Since $\mathbf{B}$ appears on both sides of (16) (both $\boldsymbol{\Lambda}$ and $\mathbf{D}$ are functions of $\mathbf{B}$ ), an iterative solution must be used to obtain the final solution. Using a combination of the steps suggested in [2]-[4] results in the iterative RCDI beamforming algorithm shown in Table I where steps 1-6 update the transmit weights while step 7 updates the receive weights using an MMSE criterion on the average signal to average interference plus noise ratio (ASAINR). Whereas the channel matrix is the only required input for the RCI algorithm, $\mathbf{S}_{\mathrm{t}, j}$ and $\mathbf{S}_{\mathrm{r}, j}$, which are nonlinear permutations of the full spatial correlation matrix for each user, are the only inputs required for the RCDI algorithm. Furthermore, the RCDI algorithm gives the same results as the 
RCI algorithm when the expectation operator is removed (i.e. $\left.\mathbf{S}_{\mathrm{t}, j}=\mathbf{H}_{j}^{T} \otimes \mathbf{H}_{j}^{H}\right)$.

Because of the nonconvex nature of the beamforming capacity expression, both RCI and RCDI algorithms only guarantee convergence to a local maximum, and they therefore may not produce the true sum capacity of the broadcast channel with linear precoding [2]. The implication of this observation is that the performance depends on the initial values of $\mathbf{D}$ and $\boldsymbol{\Lambda}$. For RCI, the beamformer created from the regularized pseudoinverse of the channel offers a good starting point. However, since an analogous initial condition for the RCDI algorithm has not yet been discovered, we will use several starting points and select the result which gives the highest bound on the average sum-rate.

The performance of using the described algorithm will be analyzed using measured channel data. While modeled channel analysis could be insightful, the difficulty lies in the fact that many models impose wide sense stationarity and spatial structure on the channel realizations, which is a characteristic that will favor CDI-based techniques especially when such techniques exploit a priori knowledge of the model. Our goal in using measured data is to show that the CDI-based schemes with reduced feedback requirements work well in realistic channels when stationarity or structure is not artificially imposed. Furthermore, multiple measurement campaigns were adopted in the simulations in order to confirm the algorithmic performance over a wide variety of spatial structures.

Fig. 5 compares the RCI result shown in Fig. 4 to the RCDI result obtained in the same Urban environment and also presents results for the Indoor and Outdoor measurements. For these simulations, it is assumed the transmitter has the full spatial correlation matrix for all users. The elements of the full correlation matrix estimated using (12) are appropriately arranged to construct the matrices $\mathbf{S}_{\mathrm{t}, j}, \mathbf{S}_{\mathrm{r}, j}$ required for the RCDI implementation. Once RCDI beamforming vectors have been found, each user is assumed to have knowledge of their transmit and receive weights which they use for all time independent of the time variations in the channel. The results in Fig. 5 confirm that while the performance of RCDI is lower than that of RCI with recent CSIT and CSIR, its throughput remains stable over large node displacements where the slight fluctuations in performance are artifacts of using measured data. The stability of RCDI implies that the feedback frequency for the RCDI algorithm is much lower than that required by the RCI approach to maintain a specified throughput. Furthermore, as shown in Fig. 5(c) for the Indoor environment, spatial structures introduced on the channel due to the environment directly affect the possibility of effectively using CDI as a precoding resource. For a spatially-white channel, the RCDI algorithm could not make any distinction between users and no gains would be possible over outdated CSI.

\section{Channel Distribution Parameterization}

The prior section demonstrated that the RCDI algorithm enables good throughput performance with reduced feedback frequency in the time-varying broadcast channel. However, because the algorithm computation requires the full spatial correlation matrix

$$
\mathbf{R}=\mathrm{E}\left[\operatorname{vec}(\mathbf{H}) \operatorname{vec}(\mathbf{H})^{H}\right]
$$

through the permutations $\mathbf{S}_{\mathrm{t}}$ and $\mathbf{S}_{\mathrm{r}}$, where the user index is dropped for convenience, the amount of data that must be fed back is significant. In fact, assuming $M$ antennas per node, each user must feedback an $M^{2} \times M^{2}$ matrix, or $M^{4}$ complex values, in contrast to the $M^{2}$ numbers that must be fed back for RCI implementation using the channel matrix. The goal of this section is to consider ways to parameterize $\mathbf{S}_{\mathrm{t}}$ and $\mathbf{S}_{\mathrm{r}}$ to reduce the required volume of feedback data. The approach taken is to explore the use of popular channel models, namely the Kronecker and Weichselberger models, which impose structure on the correlation matrix to allow its representation using smaller matrices. Throughout this development, we will make use of the properties $\mathbf{A B} \otimes \mathbf{C D}=(\mathbf{A} \otimes \mathbf{C})(\mathbf{B} \otimes \mathbf{D})$ and $(\mathbf{A} \odot \mathbf{B}) \otimes(\mathbf{C} \odot \mathbf{D})=(\mathbf{A} \otimes \mathbf{C}) \odot(\mathbf{C} \otimes \mathbf{D})$.

\section{A. Kronecker Model}

The Kronecker model [26] assumes separability between transmit and receive spatial correlation matrices. Assuming that $\mathbf{H}_{w}$ is an $M \times M$ matrix with zero-mean, unit variance, i.i.d. complex Gaussian entries, the correlated channel matrices can be realized using

$$
\mathbf{H}_{\mathrm{Kron}}=\sqrt{\mathbf{R}_{\mathrm{r}}} \mathbf{H}_{w} \sqrt{\mathbf{R}_{\mathrm{t}}}
$$

where the one-sided correlation matrices are calculated from $\mathbf{R}_{\mathrm{r}}=\mathrm{E}\left[\mathbf{H} \mathbf{H}^{H}\right]$ and $\mathbf{R}_{\mathrm{t}}=\mathrm{E}\left[\mathbf{H}^{T} \mathbf{H}^{*}\right]$ or estimated from the measured data using (10) and (11). Assuming this structure leads to the RCDI input matrix

$$
\begin{aligned}
\mathbf{S}_{\mathrm{t}}^{\mathrm{Kron}} & =\mathrm{E}\left[\mathbf{H}_{\text {Kron }}^{T} \otimes \mathbf{H}_{\text {Kron }}^{H}\right] \\
& =\mathrm{E}\left[\left(\sqrt{\mathbf{R}_{\mathrm{r}}} \mathbf{H}_{w} \sqrt{\mathbf{R}_{\mathrm{t}}}\right)^{T} \otimes\left(\sqrt{\mathbf{R}_{\mathrm{r}}} \mathbf{H}_{w} \sqrt{\mathbf{R}_{\mathrm{t}}}\right)^{H}\right] \\
& =\mathrm{E}\left[\widetilde{\mathbf{R}}_{\mathrm{t}}^{\mathrm{Kron}}\left(\mathbf{H}_{w}^{T} \otimes \mathbf{H}_{w}^{H}\right) \widetilde{\mathbf{R}}_{\mathrm{r}}^{\mathrm{Kron}}\right] \\
& =\widetilde{\mathbf{R}}_{\mathrm{t}}^{\mathrm{Kron}} \mathbf{I}_{\mathrm{t}} \widetilde{\mathbf{R}}_{\mathrm{r}}^{\text {Kron }}
\end{aligned}
$$

where $\mathbf{I}_{\mathrm{t}}=\mathrm{E}\left[\mathbf{H}_{w}^{T} \otimes \mathbf{H}_{w}^{H}\right], \widetilde{\mathbf{R}}_{\mathrm{t}}^{\text {Kron }}={\sqrt{\mathbf{R}_{\mathrm{t}}}}^{T} \otimes{\sqrt{\mathbf{R}_{\mathrm{t}}}}^{H}$, and $\widetilde{\mathbf{R}}_{\mathrm{r}}^{\text {Kron }}={\sqrt{\mathbf{R}_{\mathrm{r}}}}^{T} \otimes{\sqrt{\mathbf{R}_{\mathrm{r}}}}^{H}$. In a similar fashion we can construct

$$
\mathbf{S}_{\mathrm{r}}^{\mathrm{Kron}}=\widetilde{\mathbf{R}}_{\mathrm{r}}^{\mathrm{Kron}} \mathbf{I}_{\mathrm{r}} \widetilde{\mathbf{R}}_{\mathrm{t}}^{\mathrm{Kron}}
$$

where $\mathbf{I}_{\mathrm{r}}=\mathrm{E}\left[\mathbf{H}_{w}^{*} \otimes \mathbf{H}_{w}\right]$. These results demonstrate that the feedback information is reduced to two $M \times M$ matrices for a total of $2 M^{2}$ complex numbers.

\section{B. Rank-1 Approximation Model}

Computing $\mathbf{R}_{\mathrm{r}}$ and $\mathbf{R}_{\mathrm{t}}$ directly from the channel matrices and then using the Kronecker products to estimate the full correlation matrix can result in substantial modeling error. An alternate approach is to impose the Kronecker structure of the correlation matrix but compute estimates $\widehat{\mathbf{R}}_{\mathrm{t}}$ and $\widehat{\mathbf{R}}_{\mathrm{r}}$ from the optimization

$$
\min _{\hat{\mathbf{R}}_{\mathrm{r}}, \hat{\mathbf{R}}_{\mathrm{t}}}\left\|\mathbf{R}-\hat{\mathbf{R}}_{\mathrm{t}} \otimes \hat{\mathbf{R}}_{\mathrm{r}}\right\|^{2}
$$

These estimates can be obtained using the solution discussed in [33], which is referred to as the rank-1 approximation. These matrices can be fed back to the transmitter and used in (17) and (18). This approach also results in a feedback complexity of $2 M^{2}$ complex numbers. 
(a) Urban

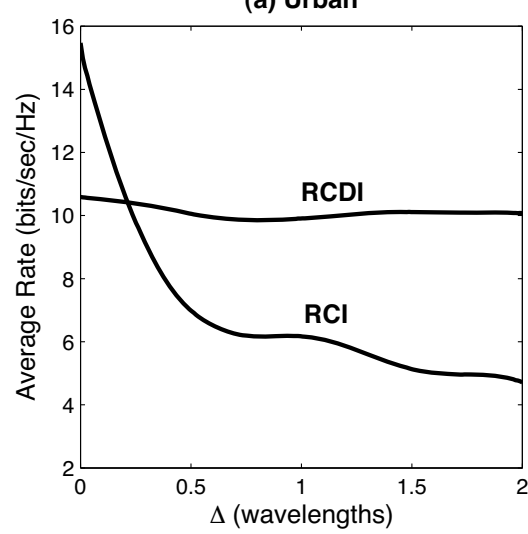

(b) Outdoor

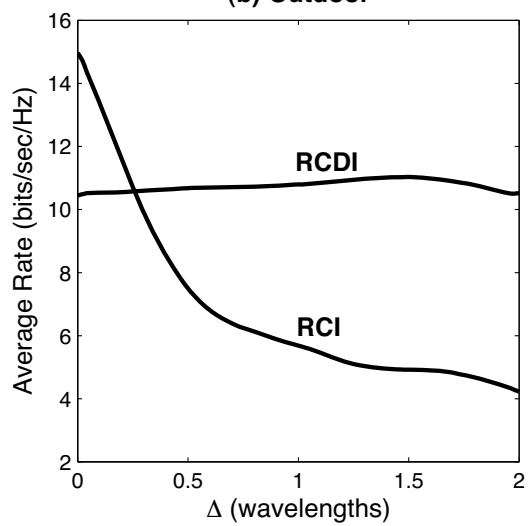

(c) Indoor

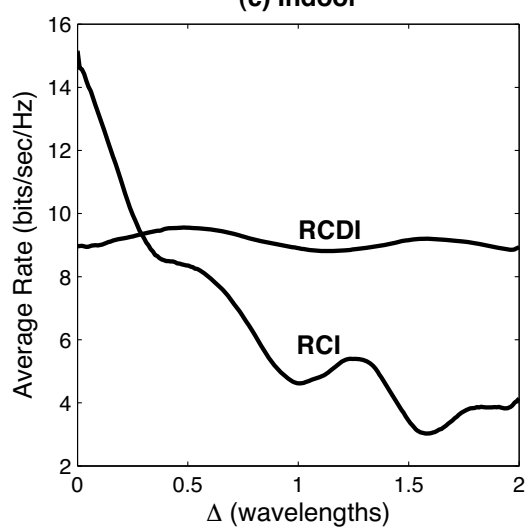

Fig. 5. Average sum-rate of RCI and RCDI versus displacement for $M=K=4$ and $P=10$. The receiver and transmitter share equally delayed knowledge of that channel given by $\Delta$ (wavelengths) from the current channel. Shown are the (a) Urban, (b) Outdoor, and (c) Indoor environments.

\section{Weichselberger Model}

The Weichselberger model [27] was introduced in an effort to overcome some of the deficiencies discovered with the Kronecker model. The Kronecker model deficiencies arise from imposing one-sided correlations on the spatial structure of the channel which result in underestimation of the channel capacity [34]. Under the Weichselberger model, channel matrix realizations are represented as

$$
\mathbf{H}_{\text {Weichs }}=\mathbf{U}_{\mathrm{r}}\left(\check{\mathbf{\Omega}} \odot \mathbf{H}_{w}\right) \mathbf{U}_{\mathrm{t}}^{T}
$$

where $\check{\mathbf{A}}$ is the element-wise square root on the matrix $A$ and the matrices $U_{r}$ and $U_{t}$ respectively contain the eigenvectors of $\mathbf{R}_{\mathrm{r}}$ and $\mathbf{R}_{\mathrm{t}}$ from the Kronecker model. Under this representation we can write

$$
\begin{aligned}
\mathbf{S}_{\mathrm{t}}^{\text {Weichs }} & =\mathrm{E}\left[\mathbf{H}_{\text {Weichs }}^{T} \otimes \mathbf{H}_{\text {Weichs }}^{H}\right] \\
& =\mathrm{E}\left[\left(\mathbf{U}_{\mathrm{r}}\left(\check{\mathbf{\Omega}} \odot \mathbf{H}_{w}\right) \mathbf{U}_{\mathrm{t}}^{T}\right)^{T} \otimes\left(\mathbf{U}_{\mathrm{r}}\left(\check{\mathbf{\Omega}} \odot \mathbf{H}_{w}\right) \mathbf{U}_{\mathrm{t}}^{T}\right)^{H}\right] \\
& =\mathrm{E}\left[\widetilde{\mathbf{U}}_{\mathrm{t}}^{\text {Weichs }}\left\{\left(\check{\boldsymbol{\Omega}} \odot \mathbf{H}_{w}\right) \otimes\left(\check{\mathbf{\Omega}} \odot \mathbf{H}_{w}\right)\right\} \widetilde{\mathbf{U}}_{\mathrm{r}}^{\text {Weichs }}\right] \\
& =\widetilde{\mathbf{U}}_{\mathrm{t}}^{\text {Weichs }}\left\{\left(\check{\boldsymbol{\Omega}}^{T} \otimes \check{\boldsymbol{\Omega}}\right) \odot \mathbf{I}_{\mathrm{t}}\right\} \widetilde{\mathbf{U}}_{\mathrm{r}}^{\text {Weichs }}
\end{aligned}
$$

where $\widetilde{\mathbf{U}}_{\mathrm{t}}^{\text {Weichs }}=\mathbf{U}_{\mathrm{t}}^{T} \otimes \mathbf{U}_{\mathrm{t}}^{H}$, and $\widetilde{\mathbf{U}}_{\mathrm{r}}^{\text {Weichs }}=\mathbf{U}_{\mathrm{r}}^{T} \otimes \mathbf{U}_{\mathrm{r}}^{H}$. Similarly,

$$
\mathbf{S}_{\mathrm{r}}^{\text {Weichs }}=\widetilde{\mathbf{U}}_{\mathrm{r}}^{\text {Weichs }}\left\{\left(\check{\boldsymbol{\Omega}}^{*} \otimes \check{\boldsymbol{\Omega}}\right) \odot \mathbf{I}_{\mathrm{r}}\right\} \widetilde{\mathbf{U}}_{\mathrm{t}}^{\text {Weichs }} \text {. }
$$

This result indicates that the RCDI implementation requires feedback of three $M \times M$ matrices for a feedback complexity of $3 M^{2}$ complex numbers. Details on estimation of these matrices from the data can be found in [27].

\section{Results}

The performance of the RCDI implementation for the different parameterizations is examined in Fig. 6 for various environments. For this plot, the power is held constant at $P=10$ while the number of antennas and users was swept assuming $M=K$. The "boxed" region highlights the values of $M$ and $K$ considered in prior Urban plots appearing in this paper. These results show that when an appropriate parameterization is used, the impact of parameterization on the overall performance is relatively small. This is noteworthy, since this parameterization significantly reduces the quantity of feedback data. As an example, for $M=6$ feedback of the full correlation matrix requires communication of 1296 complex numbers per user compared to only 108 and 72 complex numbers using parameterizations based on the Weichselberger and rank-1 models, respectively. These results also show that while the Kronecker structure for the correlation matrix is reasonable (as evidenced by the performance for the rank-1 model), it is critical that the matrices $\mathbf{R}_{\mathrm{t}}$ and $\mathbf{R}_{\mathrm{r}}$ be properly estimated. The deficiencies in the traditional Kronecker model for larger array sizes observed here appear supportive of other studies which have shown that this model fails to accurately represent the channel spatial structure as the number of antennas increases [34].

\section{CONCLUSION}

The optimal linear and nonlinear precoders for the MIMO broadcast channel result in significant throughput loss when used with outdated or erroneous CSI, and therefore require frequent feedback to maintain high performance. This paper has quantified this loss, and has adapted the sum-rate maximizing beamformer to use CDI in an effort to obtain an approach whose throughput performance remains stable in time-variant channels. Analysis of the throughput for this RCDI beamforming algorithm using measured channel data has demonstrated that it is robust to temporal variations and delay in the feedback channel and outperforms the optimal precoding techniques for node displacements that are just fractions of a wavelength. This suggests that the approach can be used to maintain good throughput with significantly reduced feedback frequency. Furthermore, simple parameterization of the channel correlation matrix using popular channel models allows significant reduction in the amount of data that must be fed back to the transmitter without resulting in significant performance loss.

\section{APPENDIX A}

\section{Broadcast Channel Per-User Mutual Information LOWER BOUND}

In order to obtain the lower bound on mutual information with measured data for the multi-user broadcast channel with 
(a) Urban

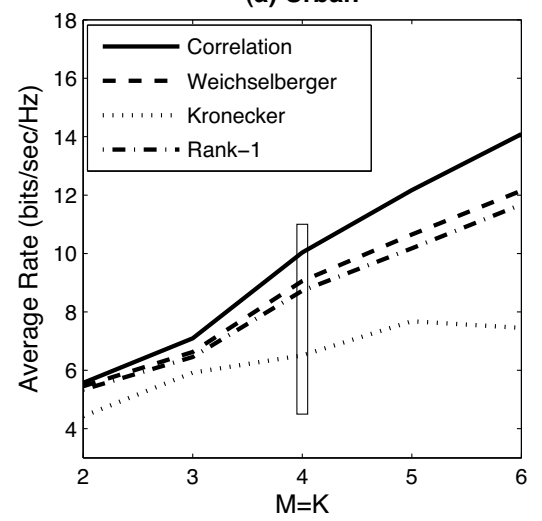

(b) Outdoor

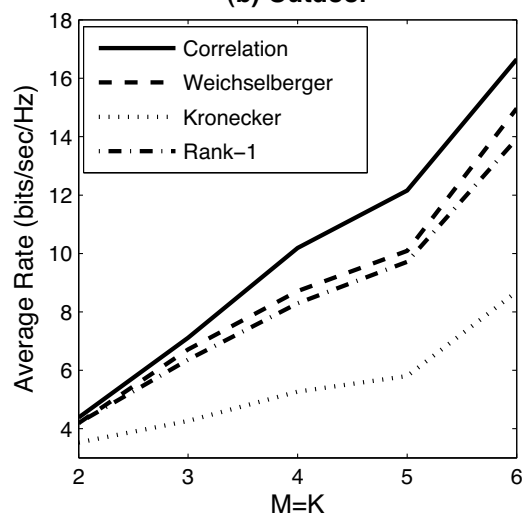

(c) Indoor

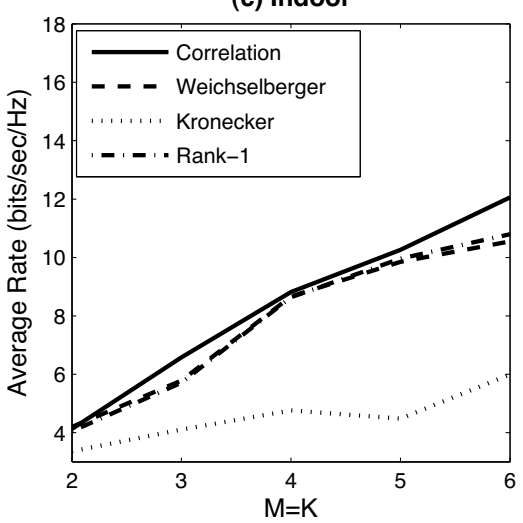

Fig. 6. Average sum-rate versus system size when the matrices $\mathbf{S}_{t}$ and $\mathbf{S}_{r}$ are generated by using: the full correlation matrix, the Weichselberger model, the Kronecker model, and a rank-1 approximation. Shown are the (a) Urban, (b) Outdoor, and (c) Indoor environments.

outdated CSIR, the proof from [29] can be modified to include multiple users with channel error that is neither i.i.d. nor zero mean. For the $j$ th user, the mutual information between input and output given the sum channel matrix and error mean is bounded by

$$
\begin{aligned}
I\left(\mathbf{x}_{j} ; \mathbf{y}_{j} \mid \widetilde{\mathbf{H}}\right) & =\mathrm{h}\left(\mathbf{x}_{j} \mid \widetilde{\mathbf{H}}\right)-\mathrm{h}\left(\mathbf{x}_{j} \mid \mathbf{y}_{j}, \widetilde{\mathbf{H}}\right) \\
& \geq \log \left|\mathbf{Q}_{j}\right|-\log \left|\mathbf{R}_{\mathbf{x}_{j}-\hat{\mathbf{x}}_{j}}\right|
\end{aligned}
$$

which assumes that $\mathbf{x}_{j}$ given $\widetilde{\mathbf{H}}_{j}$ is Gaussian with covariance $\mathbf{Q}_{j}$, the effective channel is $\widetilde{\mathbf{H}}_{j}=\hat{\mathbf{H}}_{j}+\mathbf{E}_{\mu, j}, \hat{\mathbf{x}}_{j}$ is the MMSE estimate of $\mathbf{x}_{j}, \mathrm{~h}\{\cdot\}$ is the entropy function, and $\mathbf{R}_{\mathbf{u v}}=\mathrm{E}\left[\mathbf{u v}^{H}\right]$ is the correlation matrix of the random vectors $\mathbf{u}$ and $\mathbf{v}$. The correlation matrix for the error vector can be found by

$$
\begin{aligned}
\mathbf{R}_{\mathbf{x}_{j}-\hat{\mathbf{x}}_{j}} & =\mathrm{E}\left[\left(\mathbf{x}_{j}-\hat{\mathbf{x}}_{j}\right) \mathbf{x}_{j}^{H}\right] \\
& =\mathbf{Q}_{j}-\mathbf{R}_{\mathbf{x}_{j} \mathbf{y}_{j}} \mathbf{R}_{\mathbf{y}_{j} \mathbf{y}_{j}}^{-1} \mathbf{R}_{\mathbf{y}_{j} \mathbf{x}_{j}} \\
& =\mathbf{Q}_{j}-\mathbf{Q}_{j} \widetilde{\mathbf{H}}_{j}^{H} \mathbf{R}_{\mathbf{y}_{j} \mathbf{y}_{j}}^{-1} \widetilde{\mathbf{H}}_{j} \mathbf{Q}_{j}^{H} .
\end{aligned}
$$

Using (23) with the received vector defined by (3) results is an upper-bound on the entropy expression

$$
\begin{aligned}
& \mathrm{h}\left(\mathbf{x}_{j} \mid \mathbf{y}_{j}, \widetilde{\mathbf{H}}_{j}\right) \leq \\
& \quad \log \left|\mathbf{Q}_{j}-\mathbf{Q}_{j} \widetilde{\mathbf{H}}_{j}^{H}\left(\mathbf{I}+\widetilde{\mathbf{H}}_{j} \mathbf{Q}_{j} \widetilde{\mathbf{H}}_{j}^{H}+\mathbf{Z}_{j}\right)^{-1} \widetilde{\mathbf{H}} \mathbf{Q}_{j}\right|
\end{aligned}
$$

where $\mathbf{Z}_{j}$ was defined for (4) and the notation $\mathbf{E}_{\mathrm{r}, j}=\mathbf{E}_{j}\left(\Delta_{\mathrm{r}}\right)$ and $\mathbf{E}_{\mathrm{t}, j}=\mathbf{E}_{j}\left(\Delta_{\mathrm{t}}\right)$ is used for simplicity. The matrix inversion lemma on (24) combined with (22) results in the lower bound on mutual information

$$
I_{\mathrm{DPC}}\left(\mathbf{x}_{j} ; \mathbf{y}_{j} \mid \widetilde{\mathbf{H}}_{j}\right)=\log \left|\mathbf{I}+\widetilde{\mathbf{H}}_{j}^{H}\left(\mathbf{I}+\mathbf{Z}_{j}\right)^{-1} \widetilde{\mathbf{H}}_{j} \mathbf{Q}_{j}\right| .
$$

For $K=j=1$, Eq. (25) is equivalent to the single-user bound in [29] with an effective channel $\widetilde{\mathbf{H}}$ and the given correlated error matrix.

\section{APPENDIX B}

\section{REgularized CHANNEL DistribUTION INVERSION (RCDI)}

The RCI algorithm from [2] was derived to maximize the sum-rate of a MISO broadcast channel with perfect CSI at the transmitter and receivers. This section details the steps taken to maximize an approximation of the average sumrate of a MIMO broadcast channel with perfect CDI. During the maximization process for the sum-rate bound used in the current work, the following notation is defined

$$
\begin{aligned}
\mathbf{S}_{t, j} & =\mathrm{E}\left[\mathbf{H}_{j}^{T} \otimes \mathbf{H}_{j}^{H}\right] \\
\mathbf{S}_{r, j} & =\mathrm{E}\left[\mathbf{H}_{j}^{*} \otimes \mathbf{H}_{j}\right] \\
\overline{\mathbf{H}}_{j} & =\operatorname{mat}\left(\mathbf{S}_{t, j} \operatorname{vec}\left(\mathbf{w}_{j} \mathbf{w}_{j}^{H}\right)\right) \\
\mathbf{\Lambda} & =\left[\frac{\left(\overline{\mathbf{H}}_{1} \mathbf{B}\right)_{:, 1}}{\bar{d}_{1}}, \ldots, \frac{\left(\overline{\mathbf{H}}_{K} \mathbf{B}\right)_{:, K}}{\bar{d}_{K}}\right] \\
\mathbf{D} & =\operatorname{diag}\left(\frac{\bar{n}_{1}}{\bar{d}_{1}\left(\bar{d}_{1}+\bar{n}_{1}\right)}, \ldots, \frac{\bar{n}_{K}}{\bar{d}_{K}\left(\bar{d}_{K}+\bar{n}_{K}\right)}\right)
\end{aligned}
$$

where $\operatorname{diag}(\cdot)$ returns a diagonal matrix of the input argument. In order to incorporate multiple antennas at the receivers, an effective MISO channel can be created using the beamforming vectors at each receiver. Including these values with the bound in (14) produces an effective average rate that can be written as

$$
\begin{aligned}
\bar{C} & =\sum_{j=1}^{K} \log \left(1+\frac{\mathrm{E}\left[\left|\mathbf{w}_{j}^{H} \mathbf{H}_{j} \mathbf{b}_{j}\right|^{2}\right]}{\mathrm{E}\left[\frac{\operatorname{tr}\left(\mathbf{B B}^{H}\right)}{P}+\sum_{i \neq j}\left|\mathbf{w}_{j}^{H} \mathbf{H}_{j} \mathbf{b}_{i}\right|^{2}\right]}\right) \\
& =\sum_{j=1}^{K} \log \left(1+\frac{\mathbf{B}_{:, j}^{H} \overline{\mathbf{H}}_{j} \mathbf{B}_{:, j}}{\left.\frac{\operatorname{tr}_{\left(\mathbf{B B}^{H}\right)}+\sum_{i \neq j} \mathbf{B}_{:, i}^{H} \overline{\mathbf{H}}_{j} \mathbf{B}_{:, i}}{P}\right)}\right.
\end{aligned}
$$

where $\overline{\mathbf{H}}_{j}=\operatorname{mat}\left(\mathbf{S}_{t, j} \operatorname{vec}\left(\mathbf{w}_{j} \mathbf{w}_{j}^{H}\right)\right)$ as defined in (26). Deriving the form of the RCDI beamformer follows the same formulation found in [2] for the capacity-optimal, CSI-base, RCI beamformer where, instead, the derivative of $\bar{C}$ is taken with respect to each of the transmit beamforming vector elements.

The average rate bound from (27) can be simplified and expanded as

$$
\bar{C}=\sum_{j=1}^{K} \log \left(\bar{n}_{j}+\bar{d}_{j}\right)-\log \left(\bar{d}_{j}\right)
$$

where the notation found in (14) and (26) will be used for convenience. In order to find the maximum value of $\bar{C}$ the partial derivative must be taken against each element of 
$\mathbf{B}=\left[\mathbf{b}_{1}, \ldots, \mathbf{b}_{K}\right]$. For simplicity, we show the first partial derivative of the first element; all other derivatives follow in a similar manner

$$
\begin{aligned}
\frac{\partial \bar{C}}{\partial \mathbf{B}_{1,1}} & =\frac{\bar{d}_{1}^{\prime}+\bar{n}_{1}^{\prime}}{\bar{d}_{1}+\bar{n}_{1}}-\frac{\bar{d}_{1}^{\prime}}{\bar{d}_{1}}+\left\{\frac{\bar{n}_{1}^{\prime}}{\bar{d}_{1}}-\frac{\bar{n}_{1}^{\prime}}{\bar{d}_{1}}\right\}+\sum_{i=2}^{K} \frac{\bar{d}_{i}^{\prime}+\bar{n}_{i}^{\prime}}{\bar{d}_{i}+\bar{n}_{i}}-\frac{\bar{d}_{i}^{\prime}}{\bar{d}_{i}} \\
& =\frac{\bar{n}_{1}^{\prime}}{\bar{d}_{1}}+\frac{-\bar{d}_{1}^{\prime} \bar{n}_{1}-\bar{n}_{1}^{\prime} \bar{n}_{1}}{\bar{d}_{1}\left(\bar{d}_{1}+\bar{n}_{1}\right)}-\sum_{i=2}^{K} \frac{\bar{d}_{i}^{\prime} \bar{n}_{i}}{\bar{d}_{i}\left(\bar{d}_{i}+\bar{n}_{i}\right)} \\
& =\frac{\bar{n}_{1}^{\prime}}{\bar{d}_{1}}-\sum_{i=1}^{K} \frac{\left[\mathbf{b}_{1}^{H} \overline{\mathbf{H}}_{i} \mathbf{b}_{1}\right]^{\prime} \bar{n}_{i}}{\bar{d}_{i}\left(\bar{d}_{i}+\bar{n}_{i}\right)}-\sum_{i=1}^{K} \frac{\sigma_{n}^{2} \mathbf{B}_{1,1}^{*} \bar{n}_{i}}{\bar{d}_{i}\left(\bar{d}_{i}+\bar{n}_{i}\right)} \\
& =\frac{\left(\overline{\mathbf{H}}_{1} \mathbf{B}^{*}\right)_{1,1}}{\bar{d}_{1}}-\sum_{i=1}^{K} \frac{\left(\overline{\mathbf{H}}_{i} \mathbf{B}^{*}\right)_{1,1}}{\bar{d}_{i}\left(\bar{d}_{i}+\bar{n}_{i}\right)}-\sum_{i=1}^{K} \frac{\sigma_{n}^{2} \mathbf{B}_{1,1}^{*} \bar{n}_{i}}{\bar{d}_{i}\left(\bar{d}_{i}+\bar{n}_{i}\right)}
\end{aligned}
$$

where $\bar{n}_{i}^{\prime}=0$ and $\bar{d}_{i}^{\prime}=\sigma^{2} \mathbf{B}_{1,1}^{*}+\left[\mathbf{b}_{1}^{H} \overline{\mathbf{H}}_{i} \mathbf{b}_{1}\right]^{\prime}$ for $i \neq 1$. Finding the partial derivatives for all beamforming weights, setting each equation to zero, and then stacking each solution into matrix form leads to

$$
\boldsymbol{\Lambda}-\sum_{i=1}^{K} \mathbf{D}_{i, i} \overline{\mathbf{H}}_{i} \mathbf{B}-\frac{\operatorname{tr}(\mathbf{D})}{P} \mathbf{B}=0
$$

with the final solution becoming

$$
\mathbf{B}=\left(\frac{\operatorname{tr}(\mathbf{D})}{P} \mathbf{I}+\sum_{i=1}^{i=K} \mathbf{D}_{i, i} \overline{\mathbf{H}}_{i}\right)^{-1} \boldsymbol{\Lambda} .
$$

It should be noted that, though the form of the RCI and RCDI beamformers are similar, one cannot simply take the expectation of the beamformer from [2] directly in order to find the final solution here; special care needs to be taken with the expectation operator and the random quantities found in the rate bound.

\section{REFERENCES}

[1] A. Goldsmith, S. A. Jafar, N. Jindal, and S. Vishwanath, "Capacity limits of MIMO channels," IEEE J. Selected Areas Commun., vol. 21, pp. 684-702, June 2003.

[2] M. Stojnic, H. Vikalo, and B. Hassibi, "Rate maximization in multiantenna broadcast channels with linear preprocessing," IEEE Trans. Commun., vol. 5, pp. 2338-2342, Sept. 2006.

[3] Q. H. Spencer, J. W. Wallace, C. B. Peel, T. Svantesson, A. L. Swindlehurst, and A. Gummalla, "Performance of multi-user spatial multiplexing with measured channel data," in MIMO System Technology and Wireless Communications. CRC, 2006.

[4] A. L. Anderson, J. R. Zeidler, and M. A. Jensen, "Stable transmission in the time-varying MIMO broadcast channel," EURASIP Journal on Advances in Signal Processing, vol. 2008, Article ID 617020, 14 pages, 2008. doi: $10.1155 / 2008 / 617020$.

[5] D. Gesbert, M. Kountouris, R. W. Heath, C.-B. Chae, and T. Sälzer, "Shifting the MIMO paradigm," IEEE Sig. Proc. Mag., pp. 36-46, Sept. 2007.

[6] P. Viswanath, D. Tse, and R. Laroia, "Opportunistic beamforming using dumb antennas," IEEE Trans. Inf. Theory, vol. 48, no. 6, pp. 1277-1294, June 2002.

[7] J. Chung, C.-S. Hwang, K. Kim, and Y. Kim, "A random beamforming technique in MIMO systems exploiting multiuser diversity," IEEE $J$. Selected Areas Commun., vol. 21, pp. 848-855, Jun. 2003.

[8] H. Kim, J.S. Kim, L. Jianjun, and M. Kountouris, "On the performance of limited feedback multiuser MIMO transmission in 3GPP HSDPA," Proc. 2005 IEEE 62nd Veh. Technol. Conf., vol. 1, pp. 473-476, Sept. 2005.

[9] M. Kountouris, T. Sälzer, and D. Gesbert, "Scheduling for multiuser MIMO downlink channels with ranking-based feedback," EURASIP Journal on Advances in Signal Processing, vol. 2008, Article ID 854120, 14 pages, 2008. doi:10.1155/2008/854120.
[10] D. Love, R. Heath, and T. Strohmer, "Grassmannian beamforming for multiple-input multiple-output wireless systems," IEEE Trans. Inf. Theory, vol. 49, pp. 2735-2747, Oct. 2003.

[11] C.-B. Chae, T. Inoue, R. Heath, and D. Mazzarese, "Non-iterative multiuser MIMO coordinated beamforming with limited feedforward," Proc. 2008 IEEE Intl. Conf. Acoustics, Speech, and Signal Processing, pp. 2393-2396, April 2008.

[12] H. Zheng, Y. Wu, Y. Li, S. Zhou, and J. Wang, "Limited feedback precoding scheme for downlink multiuser MIMO systems," IEICE Trans. Commun., vol. E90-B, no. 3, pp. 689-692, Mar. 2007.

[13] P. Ding, D. J. Love, and M. D. Zoltowski, "Multiple antenna broadcast channels with shape feedback and limited feedback," IEEE Trans. Signal Processing, vol. 55, pp. 3417-3428, July 2007.

[14] N. Jindal, "MIMO broadcast channels with finite-rate feedback," IEEE Trans. Inf. Theory, vol. 52, no. 11, pp. 5045-5060, Nov. 2006.

[15] G. Caire, N. Jindal, M. Kobayashi, and N. Ravindran, "Quantized vs. analog feedback for the MIMO broadcast channel: A comparison between zero-forcing based achievable rates," in Proc. 2007 IEEE Intl. Symp. on Info. Theory, June 2007.

[16] W. Cheng and R. Murch, "MU-MISO transmission with limited feedback," IEEE Trans. Wireless Commun., vol. 6, no. 11, pp. 39073913, Nov. 2007.

[17] T. Yoo, N. Jindal, and A. Goldsmith, "Multi-antenna downlink channels with limited feedback and user selection," IEEE J. Selected Areas Commun., vol. 25, pp. 1478-1491, Sept. 2007.

[18] R. Zhang, J. Cioffi, and Y. Liang, "Throughput comparison of wireless downlink transmission schemes with multiple antennas," in Proc. 2005 IEEE Intl. Conf. Commun., May 2005.

[19] M. Cho, Y. Kim, Yo. Kim, and D. Hong, "An efficient CQI feedback scheme for multiuser MIMO systems," Proc. 2007 IEEE 66nd Veh. Technol. Conf., pp. 625-629, Oct 2007.

[20] A. Soysal and S. Ulukus, "Optimum power allocation for single-user MIMO and multi-user MIMO-MAC with partial CSI," IEEE J. Selected Areas Commun., vol. 25, pp. 1402-1412, Sept. 2007.

[21] V. Raghavan, A. Sayeed, and V. Veeravalli, "Limited feedback precoder design for spatially correlated MIMO channels," Information Sciences and Systems, 2007. CISS '07. 41st Annual Conference on, pp. 113-118, March 2007.

[22] M. Kountouris, D. Gesbert, and L. Pittman, "Transmit correlation-aided opportunistic beamforming and scheduling," in Proc. Europ. Signal Processing Conf., Sept. 2006.

[23] D. Hammarwall, M. Bengtsson, and B. Ottersten, "Acquiring partial CSI for spatially selective transmission by instantaneous channel norm feedback," IEEE Trans. Signal Processing, vol. 56, no. 3, pp. 11881204, Mar. 2008.

[24] J. W. Wallace, M. A. Jensen, A. L. Swindlehurst, and B. D. Jeffs, "Experimental characterization of the MIMO wireless channel: Data acquisition and analysis," IEEE Trans. Wireless Commun., vol. 2, pp. 335-343, Mar. 2003.

[25] J. W. Wallace and M. A. Jensen, "Time varying MIMO channels: Measurement, analysis, and modeling," IEEE Trans. Antennas Propag., vol. 54, pp. 3265-3273, Nov. 2006.

[26] S. Shiu, G. J. Foschini, M. J. Gans, and J. M. Khan, "Fading correlation and its effect on the capacity of multielement antenna systems," IEEE Trans. Commun., vol. 48, pp. 502-513, Mar. 2000.

[27] W. Weichselberger, M. Herdin, H. Özcelik, and E. Bonek, "A stochastic MIMO channel model with joint correlation of both link ends," IEEE Transactions on Wireless Communications, vol. 5, no. 1, pp. 90-99, 2006.

[28] S. Yang and J. Belfiore, "The impact of channel estimation error on the DPC region of the two-user Gaussian broadcast channel," in Proc. of the 43rd Allerton Conf. on Comm., Control, and Comp., Sept. 2005.

[29] T. Yoo and A. Goldsmith, "Capacity and power allocation for fading MIMO channels with channel estimation error," IEEE Trans. Inf. Theory, vol. 52, pp. 2203-2214, May 2006.

[30] B. T. Maharaj, J. W. Wallace, and M. A. Jensen, "A low-cost openhardware wideband multiple-input multiple-output (MIMO) wireless channel sounder," Submitted to the IEEE Trans. on Instrum. Meas.

[31] J. W. Wallace, M. A. Jensen, A. Gummalla, and H. Lee, "Experimental characterization of the outdoor MIMO wireless channel temporal variation," IEEE Trans. Veh. Technol., vol. 56, pp. 1041-1049, May 2007.

[32] V. Anreddy and M. A. Ingram, "Capacity of measured Ricean and Rayleigh indoor MIMO channels at $2.4 \mathrm{GHz}$ with polarization and spatial diversity," in Proc. IEEE Wireless Communications \& Networking Conference (WCNC), April 2006.

[33] C. F. Van Loan and N. Pitsianis, "Approximations with Kronecker products," in Linear Algebra for Large Scale and Real Time Applications, 
M. S. Moonen and G. H. Golub, Eds. Kluwer Academic Publishers, 1993.

[34] H. Ozcelik, M. Herdin, J. Wallace, and E. Bonek, "Deficiencies of 'Kronecker' MIMO radio channel model," Electronics Letters, vol. 39, pp. 1209-1210, Aug. 2003.

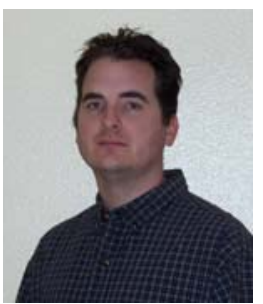

Adam L. Anderson (S'00) received B.S. (2002) and MS. (2004) degrees in electrical engineering from Brigham Young University, Provo, UT, and is currently working toward a Ph.D. degree in electrical engineering at UCSD. His current research interests focus on MIMO systems in mobile ad hoc networks.

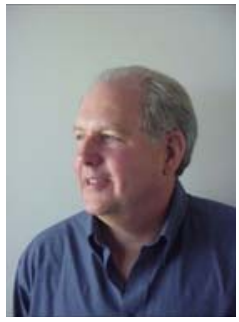

James R. Zeidler (M'76, SM'84, F'94) is a research scientist/senior lecturer in the Department of Electrical Engineering at the University of California, San Diego. He is a faculty member of the UCSD Center for Wireless Communications and the University of California Institute for Telecommunications and Information Technology. He has more than 200 technical publications and thirteen patents for communication, signal processing, data compression techniques, and electronic devices. Dr. Zeidler was elected Fellow of the IEEE in 1994 for his technical contributions to adaptive signal processing and its applications. He received a Frederick Ellersick award from the IEEE Communications Society at the IEEE Military Communications Conference in 1995, the Navy Meritorious Civilian Service Award in 1991, and the Lauritsen-Bennett Award for Achievement in Science from the Space and Naval Warfare Systems Center in 2000. He was an Associate Editor of the IEEE Transactions on Signal Processing.

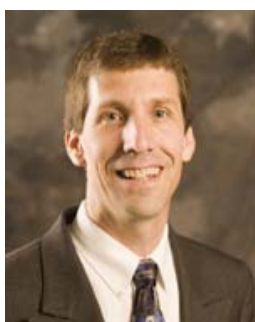

Michael A. Jensen (S'93, M'95, SM'01, F'08) received the B.S. (summa cum laude) and M.S. degrees in Electrical Engineering from Brigham Young University (BYU) in 1990 and 1991, respectively, and the Ph.D. in Electrical Engineering at the University of California, Los Angeles (UCLA) in 1994. Since 1994, he has been at the Electrical and Computer Engineering Department at BYU where he is currently a Professor and Department Chair. His research interests include antennas and propagation for communications, microwave circuit design, numerical electromagnetics, and optical fiber communications. He currently chairs the Joint Meetings Committee for the IEEE Antennas and Propagation Society and previously served as a member of the Administrative Committee for this same society and as associate editor for the IEEE Transactions on Antennas and Propagation. He has also served as Vice-Chair or Technical Program Chair for four different symposia. He was awarded the H. A. Wheeler paper award in the IEEE Transactions on Antennas and Propagation in 2002, and the best student paper award at the 1994 IEEE International Symposium on Antennas and Propagation. 